\title{
SOME PROPERTIES OF PARTIAL SUMS OF THE HARMONIC SERIES
}

\author{
PAUL ERDÖS AND IVAN NIVEN
}

It has been proved that $\sum_{k=m}^{n} k^{-1}$ cannot be an integer ${ }^{1}$ for any pair of positive integers $m$ and $n$. More generally, $\sum_{k=0}^{n}(m+k d)^{-1}$ cannot be an integer. ${ }^{2}$ We prove two theorems of a similar nature.

THEOREM 1. There is only a finite number of integers $n$ for which one or more of the elementary symmetric functions of $1,1 / 2,1 / 3, \cdots, 1 / n$ is an integer.

PRoof. Let $\sum_{k, n}$ denote the $k$ th symmetric function of $1,1 / 2,1 / 3$, $\cdots, 1 / n$. Since each term of $\sum_{k, n}$ is contained $k$ ! times in the expansion of $(1+1 / 2+\cdots+1 / n)^{k}$, we have, for $k>3 \log n$ and $n$ sufficiently large,

$$
\sum_{k, n}<\frac{(1+1 / 2+\cdots+1 / n)^{k}}{k !}<\frac{(1+\log n)^{k}}{k !}<1,
$$

where the second inequality arises from the usual comparison of $\log n$ with the harmonic series, and the third inequality is implied by the hypothesis $k>3 \log n$.

Henceforth we take $k<3 \log n$. By a theorem of A. E. Ingham ${ }^{3}$ there is a prime between $x$ and $x+x^{5 / 8}$. This implies that there is a prime $p$ between $1+n /(k+1)$ and $n / k$ for $k<3 \log n$ and $n$ sufficiently large. Hence $\sum_{k, n}$ contains the term

$$
\frac{1}{p} \cdot \frac{1}{2 p} \cdots \frac{1}{k p}=\frac{1}{k ! p^{k}} .
$$

Now $(k !, p)=1$ since $k<n /(k+1)$, and hence no other term in $\sum_{k, n}$ has a denominator divisible by $p^{k}$. So if $\sum_{k, n}=a / b$, we know that $p^{k} \mid b$ and $p \nmid a$, which proves the theorem.

By a similar but more complicated argument we can prove the same

Received by the editors November 5,1945 .

1 Cf. Polya-Szegö, Aufgaben und Lehrsattze aus der Analysis, vol. 2, Berlin, 1925, chap. 8, p. 159, problem 250.

${ }^{2}$ Cf. T. Nagell, Eine Eigenschaft gewissen Summen, Skrifter Oslo, no. 13 (1923) pp. 10-15.

${ }^{8}$ On the difference between consecutive primes, Quart. J. Math. Oxford Ser. vol. 8 (1937) p. 256. This result is actually stronger than necessary for our use here. The classical estimates will suffice. 
result for the elementary symmetric functions of $1 / m, 1 /(m+1)$, $\cdots, 1 / n$, and of $1 / m, 1 /(m+d), 1 /(m+2 d), \cdots, 1 /(m+n d)$.

It should be noted that $\sum_{2,3}$ is an integer; we know of no other integral case. Theorem 1 can be proved without the use of the prime number theorem, and this proof could be used to determine the bound on $n$, above which the result of the theorem holds. For smaller values of $n, \sum_{k, n}$ could be checked, but the proof is complicated and the limits would be large.

THEOREM 2. No two partial sums of the harmonic series can be equal; that is, it is not possible that

$$
\begin{aligned}
1 / m+1 /(m+1)+\cdots+ & 1 / n \\
& =1 / x+1 /(x+1)+\cdots+1 / y .
\end{aligned}
$$

Proof. We assume that $n<x$. Clearly if (1) has a solution, then any prime divisor of one of the denominators must divide another. Hence by Bertrand's postulate we can be certain that $y<2 x-1$, since otherwise a prime $p>n$ would be one of the denominators on the right side of (1).

LEMMA. Any solution of (1) must satisfy $y<x+x^{1 / 2}-1$.

To prove this we use a theorem of Sylvester and Schur which states that if $n>k$, then in the set $n, n+1, \cdots, n+k-1$ there is an integer containing a prime divisor greater than $k$. In our case $x>y-x+1$, so that there is a prime $p>y-x+1$ which divides one and only one (say $a p$ ) of the integers $x, x+1, x+2, \cdots, y$. Also $p$ must divide one (say $b p$ ) of the set $m, m+1, m+2, \cdots, n$, and certainly not more than one, since $n-m<y-x$. Then $1 / a p$ and $1 / b p$ are the only terms in equation (1) whose denominators are divisible by $p$, and since

$$
1 / b p-1 / a p=(a-b) / a b p,
$$

we conclude that $p$ must divide $a-b$, whence $a-b \geqq p$ and $a \geqq p+1$. This implies that

$$
y \geqq a p \geqq p^{2}+p>(y-x+1)^{2}+y-x+1
$$

or

$$
x-1>(y-x+1)^{2},
$$

which proves the lemma.

Next we obtain estimates for the expressions in (1). First we note that

4 Cf. Paul Erdös, J. London Math. Soc. vol. 9 (1934) p. 282. 


$$
\begin{aligned}
\log \frac{2 k+1}{2 k-1} & =\log \left(1+\frac{1}{2 k}\right)-\log \left(1-\frac{1}{2 k}\right) \\
& =\frac{1}{k}+\sum_{j=1}^{\infty} \frac{2}{(2 j+1)(2 k)^{2 j+1}} .
\end{aligned}
$$

Solving for $1 / k$, and summing the result for $k=m, m+1, \cdots, n$, we obtain

$$
\begin{aligned}
\frac{1}{m}+\frac{1}{m+1}+\cdots & +\frac{1}{n} \\
& =\log \frac{2 n+1}{2 m-1}-\sum_{k=m}^{n} \sum_{j=1}^{\infty} \frac{2}{(2 j+1)(2 k)^{2 j+1}},
\end{aligned}
$$

and similarly

$$
\begin{aligned}
\frac{1}{x}+\frac{1}{x+1}+\cdots & +\frac{1}{y} \\
& =\log \frac{2 y+1}{2 x-1}-\sum_{k=x}^{y} \sum_{j=1}^{\infty} \frac{2}{(2 j+1)(2 k)^{2 j+1}} .
\end{aligned}
$$

Now (1) and our assumption that $n<x$ imply that for any $j \geqq 1$,

$$
\sum_{k} \frac{2}{(2 j+1)(2 k)^{2 j+1}}
$$

is greater when summed over $k=m, m+1, \cdots, n$ than over $k=x$, $x+1, \cdots, y$ and so, comparing the right sides of (2) and (3), we see that

$$
(2 n+1) /(2 m-1)>(2 y+1) /(2 x-1) .
$$

Thus, ignoring the sum on the right side of (3), we may write

$$
\log \frac{(2 n+1)(2 x-1)}{(2 m-1)(2 y+1)}<\sum_{k=m}^{n} \sum_{j=1}^{\infty} \frac{2}{(2 j+1)(2 k)^{2 j+1}} .
$$

The infinite sum on the right can be replaced by $4 / 3$ times the first term, since each term is more than 4 times the next. The numerator of the fraction on the left exceeds the denominator by at least 2 , since both are odd, and hence the left side exceeds

$$
\log \left(1+\frac{2}{(2 m-1)(2 y+1)}\right)>\frac{1}{(2 m-1)(2 y+1)} .
$$

Thus we have 
(5) $\frac{1}{(2 m-1)(2 y+1)}<\sum_{k=m}^{n} \frac{2 \cdot 4 / 3}{3(2 k)^{3}}<\frac{1}{9 m^{2}} \sum_{k=m}^{n} \frac{1}{k}=\frac{1}{9 m^{2}} \sum_{k=x}^{y} \frac{1}{k}$.

But the last sum has fewer than $x^{1 / 2}$ terms (by the lemma) and each term is not greater than $1 / x$. And since $(2 m-1)(2 y+1)<4 m y$, inequality (5) implies that

or

$$
\frac{1}{4 m y}<\frac{1}{9 m^{2}} \cdot \frac{x^{1 / 2}}{x}
$$

(6)

$$
9 m x^{1 / 2}<4 y .
$$

But also $1 / m \leqq 1 / m+\cdots+1 / n<x^{1 / 2} \cdot(1 / x)=1 / x^{1 / 2}$, so that $x^{1 / 2}<m$, which together with (6) implies that $9 x<4 y$, which contradicts the lemma. This completes the proof of Theorem 2 .

In conclusion, we observe that $1 / 2+1 / 3+1 / 4 \equiv 1 / 12(\bmod 1)$. Whether the sums in equation (1) are congruent $(\bmod 1)$ for infinitely many values $m, n, x, y$ is an unsolved problem.

STANFORD UNIVERSITY AND

Purdue University 